\title{
NMR elastometry of fluid membranes in the mesoscopic regime
}

\author{
Gary V. Martinez, ${ }^{1}$ Emily M. Dykstra, ${ }^{1}$ Silvia Lope-Piedrafita, ${ }^{2}$ Constantin Job, ${ }^{1}$ and Michael F. Brown ${ }^{1, *}$ \\ ${ }^{1}$ Department of Chemistry, University of Arizona, Tucson, Arizona 85721 \\ ${ }^{2}$ Department of Physics, University of Arizona, Tucson, Arizona 85721
}

(Received 3 January 2002; revised manuscript received 21 May 2002; published 27 November 2002)

\begin{abstract}
In solid-state ${ }^{2} \mathrm{H}$ NMR of fluid lipid bilayers, quasielastic deformations at MHz frequencies are detected as a square-law dependence of the nuclear spin-lattice $\left(R_{1 Z}\right)$ relaxation rates and order parameters $\left(S_{\mathrm{CD}}\right)$. The signature square-law slope is found to decrease progressively with the mole fraction of cholesterol and with the acyl chain length, due to a stiffening of the membrane. The correspondence to thermal vesicle fluctuations and molecular dynamics simulations implies that a broad distribution of modes is present, ranging from the membrane size down to the molecular dimensions.
\end{abstract}

DOI: 10.1103/PhysRevE.66.050902

PACS number(s): 87.16.Dg, 87.15.Kg, 82.56.-b

Elastic deformation of membranes plays a central role in biological physics with regard to lipid-protein interactions, domain (raft) formation, and various nano- and microstructures implicated in cellular functions [1]. In this Rapid Communication, we describe nuclear magnetic resonance (NMR) studies of the influences of cholesterol as well as the membrane thickness on material properties of lipid bilayers. We have found a surprisingly good correspondence of the analysis of atom-specific NMR data using a simple continuum theory with the macroscopic bending moduli of the same systems, involving longer length scales. A hierarchy of quasielastic membrane disturbances is evident, with wavelengths spanning the macroscopic bilayer size down to the microscopic dimensions of the flexible lipid molecules [2].

Apart from their biological roles, membrane lipids present fundamental issues of broad interest to physicists [3]. Generally there are two avenues to investigating the bilayer elasticity. Either one can subject the bilayer to micromechanical deformation [4], or the thermal fluctuations of the system can be observed [5-7]. In each case, the bilayer is described by the same material constants. The issue of how collective fluctuations of liquid-crystalline membranes are related to atomic-level motions can also be explored by molecular dynamics (MD) simulations [8,9]. A complementary framework [10] involves the application of differential geometry, together with the continuum theory of elasticity [11]. Although the two approaches have had considerable success, their unification requires further effort on both the experimental and the theoretical side.

Our results support the view that NMR measurements of soft membranes detect collective properties of the molecules in the mesoscopic regime $[2](\approx 1-100 \mathrm{~nm})$. Solid-state NMR spectroscopy depends on microscopic observables, yet is characterized by a relatively long time scale $\left(\approx 10^{-6} \mathrm{~s}\right)$, which involves a distribution over atomic and molecular sites $[2,12]$. It has the potential for bridging the gap between the microscopic domain $(\lesssim 1 \mathrm{~nm})$, involving the constituent molecules, and the macroscopic bulk material ( $\gtrsim 500 \mathrm{~nm})$. In deuterium NMR of membrane lipids, the quadrupolar coupling of the ${ }^{2} \mathrm{H}$ nucleus ( $\operatorname{spin} I=1$ ) with the local electric

\footnotetext{
*Author to whom correspondence should be addressed.
}

field gradient (EFG) of the $\mathrm{C}-{ }^{2} \mathrm{H}$ bond is investigated $[12,13]$. Studies of lipids having perdeuterated acyl chains allow the entire membrane hydrocarbon interior to be probed [13]. For bilayers of ${ }^{2} \mathrm{H}$-labeled lipids in the fluid state, the residual quadrupolar couplings manifest the segmental order parameters $\left(S_{\mathrm{CD}}\right)$, which characterize the dynamic bilayer structure. The spin-lattice relaxation rates $\left(R_{1 Z}\right)$ describe recovery of the energy level populations of the ${ }^{2} \mathrm{H}$ nuclei after an inverting $(\pi)$ pulse, and indicate the types of lipid motions that average the EFG tensor. Such motions can be due to local segmental isomerizations of the flexible lipids, effective molecular rotations within the potential of mean force, and/or collective excitations of the bilayer.

Figure 1(a) depicts an inversion-recovery series of ${ }^{2} \mathrm{H}$ NMR spectra for a random dispersion of DMPC- $d_{54} /$ cholesterol $(1: 1)$ in the liquid-ordered phase. A superposition of Pake doublets [12] corresponding to the various motionally inequivalent $\mathrm{C}-{ }^{2} \mathrm{H}$ bonds is evident. The sharp spectral edges represent the weak singularities due to the $\theta=90^{\circ}$ orientation of the bilayer normal (director axis) relative to the main magnetic field, and the weak shoulders are due to $\theta$ $=0^{\circ}$. The ${ }^{2} \mathrm{H}$ NMR spectra indicate a single bilayer environment on the time scale of $\approx 100 \mu \mathrm{s}$; no evidence for longerlived cholesterol-containing complexes or domains is found. In Fig. 1(b) the partially-relaxed ${ }^{2} \mathrm{H}$ NMR spectra are deconvolved [12] to yield spectra corresponding to the $\theta=0^{\circ}$ orientation. The increased resolution allows a more accurate determination of the $R_{1 Z}$ rates. After the inverting $\pi$ pulse, the $z$-magnetization appears negative, and recovers to the equilibrium positive signal. Surprisingly, the recovery is faster as the residual quadrupolar splittings increase, rather than decreasing as expected for a reduction in motional mean-squared amplitudes. This paradox is most readily explained by order fluctuations involving modulation of a preaveraged or residual coupling tensor, rather than local segmental motions.

Here we show that comparative ${ }^{2} \mathrm{H}$ NMR relaxation studies of fluid lipid bilayers as a function of sterol content and bilayer thickness closely parallel studies of macroscopic bilayers, and that analogous material constants can be estimated. Micropipette deformation [4,12] and shape fluctuation analysis $[6,7]$ of giant lipid vesicles have revealed that 


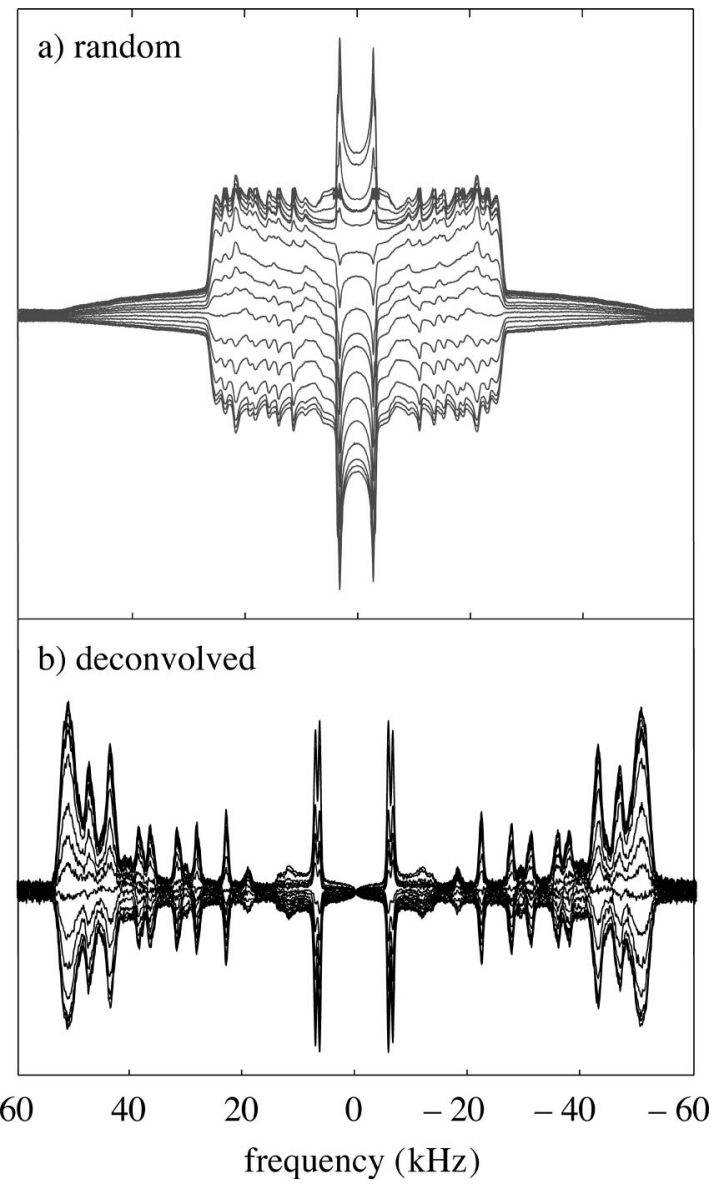

FIG. 1. Inversion recovery of ${ }^{2} \mathrm{H}$ nuclear magnetization for a random DMPC- $d_{54} /$ cholesterol (1:1) multilamellar dispersion in the liquid-ordered phase at $T=44{ }^{\circ} \mathrm{C}$. Panel (a) shows the random, partially relaxed ${ }^{2} \mathrm{H}$ NMR spectra, and panel (b) the deconvolved spec$\operatorname{tra}\left(\theta=0^{\circ}\right)$. The sample contained $20 \mathrm{mM}$ Tris buffer at $\mathrm{pH} 7.3(50$ wt. $\% \mathrm{H}_{2} \mathrm{O}$ ). Data were acquired at $76.8 \mathrm{MHz}$ using a phase-cycled, inversion-recovery quadrupolar echo pulse sequence, $\pi-t_{1}$ $-(\pi / 2)_{x}-\tau-(\pi / 2)_{y}-t_{2}$ (acquire), where $t_{1}$ is a variable delay ranging from $5 \mathrm{~ms}$ (bottom) to $3 \mathrm{~s}$ (top).

cholesterol produces a dramatic stiffening of the membrane. We wanted to establish whether this stiffening is manifested at the mesoscopic level of the $R_{1 Z}$ measurements at $\mathrm{MHz}$ frequencies. Profiles of the order parameters and relaxation rates as a function of acyl segment position $(i)$ are shown in Figs. 2(a) and 2(b), respectively, for DMPC- $d_{54}$ containing cholesterol in the liquid-ordered [12] phase, where the assignments follow Ref. [14]. The segmental order parameters $S_{\mathrm{CD}}^{(i)}$ of the various $\mathrm{C}-{ }^{2} \mathrm{H}$ bonds were obtained using $\left|\Delta \nu_{Q}^{(i)}\right|=\frac{3}{2} \chi_{Q}\left|S_{\mathrm{CD}}^{(i)}\right|\left|P_{2}(\cos \theta)\right|$ where $\Delta \nu_{Q}^{(i)}$ is the $i$ th residual quadrupolar splitting, $\chi_{Q}$ is the static quadrupolar coupling constant $(170 \mathrm{kHz})$, and $P_{2}(\cos \theta) \equiv 1 / 2\left(3 \cos ^{2} \theta-1\right)$. For each of the resolved peaks in the de-Paked ${ }^{2} \mathrm{H}$ NMR spectra, the $R_{1 Z}$ rates were calculated from non-linear regression fits to the recovery curves [14]. The order profiles in Fig. 2(a) include a plateau, followed by a reduction in orientational order within the central hydrocarbon region of the bilayer [13], which is explained by end effects of the acyl chains. Upon addition of cholesterol, the order parameters increase

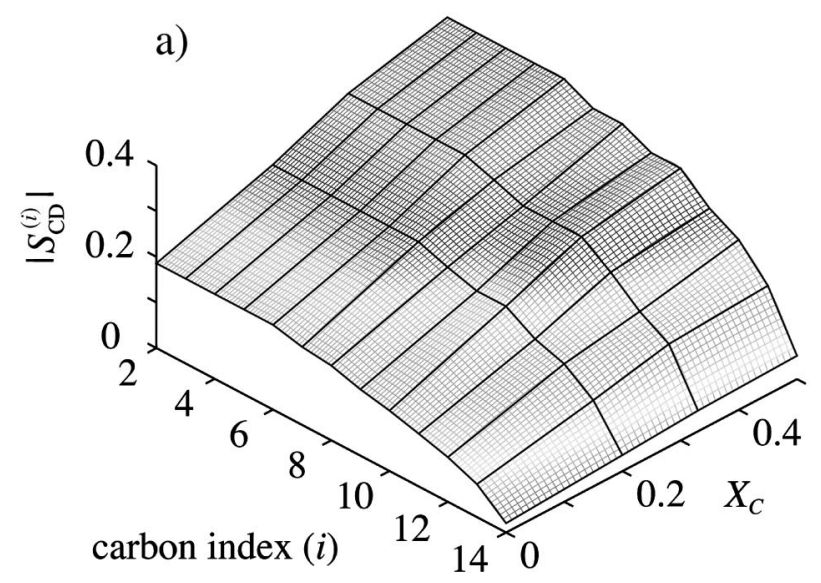

b)

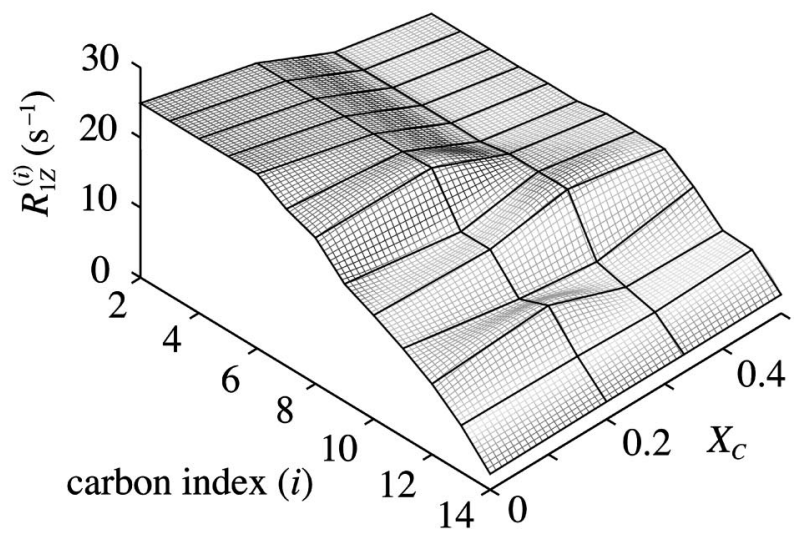

FIG. 2. Profiles of ${ }^{2} \mathrm{H}$ NMR experimental observables at 76.8 $\mathrm{MHz}$ versus acyl position for mixtures of DMPC- $d_{54} /$ cholesterol at $T=44{ }^{\circ} \mathrm{C}$. In panel (a) the orientational order parameters $\left|S_{\mathrm{CD}}^{(i)}\right|$ are plotted against the carbon index $(i)$ for various mole fractions of cholesterol $\left(X_{C}\right)$. Panel (b) shows the corresponding $R_{1 Z}^{(i)}$ relaxation data.

due to a reduction of the lateral membrane area $\langle A\rangle$ and an increase in hydrocarbon thickness $D_{C}$. In Fig. 2(b) the $R_{1 Z}$ profiles bear a striking resemblance to the corresponding order profiles, yet with a stronger dependence on acyl position. Moreover, as the mole fraction of cholesterol $X_{C}$ in the lipid mixtures is increased, the $R_{1 Z}$ rates decrease, which is exactly opposite to the effect of cholesterol on the order profiles in Fig. 2(a).

Clearly such behavior would be difficult to rationalize in the absence of an appropriate conceptual framework. By combining the ${ }^{2} \mathrm{H}$ NMR relaxation data with the order parameters, the results can give a more complete picture of the biophysical properties of membrane lipids. The segmental order parameters are defined as

$$
S_{\mathrm{CD}}^{(i)} \equiv \frac{1}{2}\left\langle 3 \cos ^{2} \beta^{(i)}-1\right\rangle,
$$

in which $\beta^{(i)}$ is the angle between the $i$ th $\mathrm{C}-{ }^{2} \mathrm{H}$ bond and the director, and the angular brackets pertain to a time or ensemble average. The NMR relaxation rates allow one to disentangle the motions in the $\mathrm{MHz}$ range giving rise to $S_{\mathrm{CD}}$. 
They depend on the mean-squared amplitudes of the fluctuations of the $\mathrm{C}-{ }^{2} \mathrm{H}$ labeled molecules, and the associated correlation times. In terms of theory [13], $R_{1 Z}$ describes coupling of the ${ }^{2} \mathrm{H}$ nuclear spins to the lattice (surroundings) within the weak collision regime, and is given by

$$
R_{1 Z}=\frac{3}{4} \pi^{2} \chi_{Q}^{2}\left[J_{1}\left(\omega_{0}\right)+4 J_{2}\left(2 \omega_{0}\right)\right]
$$

where $\omega_{0}$ is the nuclear Larmor frequency. The symbols $J_{m}(\omega)=\int G_{m}(t) \exp (-i \omega t) d t$ denote the (irreducible) spectral densities of motion, in which $G_{m}(t)$ are temporal autocorrelation functions (rank-2) of the perturbing Hamiltonian $(m=1,2)$.

Equation (2) illustrates how nuclear spin relaxation measurements are connected to the membrane dynamics in terms of the power spectra of lipid motions that produce averaging of the coupling tensor. The rates manifest the strength of the $\mathrm{C}-{ }^{2} \mathrm{H}$ bond fluctuations as a function of frequency, and are related to the softness of the membrane. A model-free, square-law functional dependence of the longitudinal $R_{1 Z}^{(i)}$ rates and $S_{\mathrm{CD}}^{(i)}$ order parameters is often found for the resolved splittings of phospholipid bilayers with ${ }^{2} \mathrm{H}$-labeled saturated acyl chains [2]. This dependence on the motional amplitudes signifies relatively slow bilayer motions that modulate the residual coupling tensors left over from faster segmental motions. Given a simple composite membrane deformation model [15], the $R_{1 Z}$ rates are due to a broad spectrum of 3D collective bilayer excitations [2], with effective rotations of the lipids. Transverse ${ }^{2} \mathrm{H}$ NMR spin relaxation studies also provide evidence for $2 \mathrm{D}$ collective motions of the membrane film at lower frequencies [16,17]. By contrast, local trans-gauche isomerizations along the chains modulate the same static NMR coupling tensor, and do not yield such a square-law.

Double-logarithmic plots of $R_{1 Z}^{(i)}$ against $\left|S_{\mathrm{CD}}^{(i)}\right|$ are summarized in Fig. 3(a) for three different mole ratios of the DMPC- $d_{54} /$ cholesterol bilayers. The approximately linear region has a slope of nearly $n=2$, consistent with a simple square-law functional dependence of $R_{1 Z}^{(i)}$ on $\left|S_{\mathrm{CD}}^{(i)}\right|$. Such square-law plots for each of the resolved quadrupolar splittings are shown in Fig. 3(b) for the multilamellar dispersions of DMPC- $d_{54} /$ cholesterol. (Note that the individual acyl positions are not distinguished.) As the cholesterol mole fraction $X_{C}$ increases, the slopes of the plots are dramatically reduced. From studies of micromechanical deformation [12] and thermal fluctuations [6,7] of giant lipid vesicles, it is known that cholesterol stiffens the membrane considerably. Thus it is plausible that we are seeing with NMR relaxation the same phenomena. Interestingly, for all the systems studied the ordinate intercepts are nearly alike, suggesting the local segmental motions of the flexible lipids are comparable, in contrast to the longer wavelength quasielastic modes.

In the limit of short wavelength excitations $\approx$ the bilayer thickness and less, the spectral density is [15]

$$
J_{m}(\omega)=\frac{5}{2} S_{\mathrm{CD}}^{2} D \omega^{-(2-d / 2)}\left[\left|D_{-1 m}^{(2)}(\theta)\right|^{2}+\left|D_{1 m}^{(2)}(\theta)\right|^{2}\right] .
$$
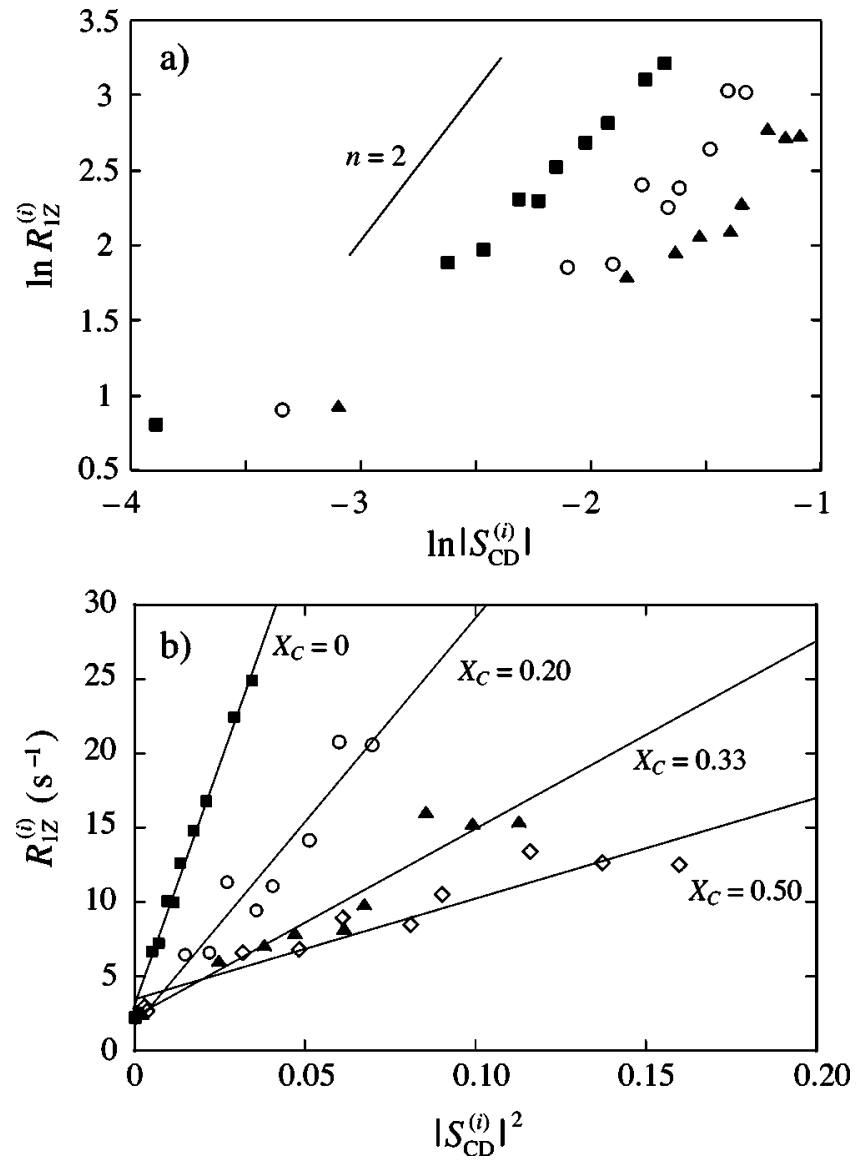

FIG. 3. Functional dependence of relaxation rate $R_{1 Z}^{(i)}$ and order parameter $\left|S_{\mathrm{CD}}^{(i)}\right|$ profiles at $76.8 \mathrm{MHz}$ for DMPC- $d_{54} /$ cholesterol mixtures at $T=44{ }^{\circ} \mathrm{C}$. Panel (a) shows logarithmic plots for three different cholesterol mole fractions $X_{C}$. The data are compared to the slope of $n=2$ predicted for order fluctuations in the limit of a small constant contribution. In panel (b) a square-law functionality of the corresponding relaxation and order profiles is assumed for the different values of $X_{C}$.

Here $\omega$ is the angular frequency, $D$ is the viscoelastic constant, $d$ is the dimensionality, and $\mathbf{D}^{(2)}$ indicates the secondrank Wigner rotation matrix. The irreducible spectral densities $J_{m}(\omega)$ depend on the square of the observed order parameter $S_{\mathrm{CD}}$, and the slope of a square-law plot is inversely related to the softness of the membrane. For 3D quasielastic fluctuations [2,15], the viscoelastic constant is given by $D=3 k_{B} T \sqrt{\eta} / 5 \pi \sqrt{2 K^{3}} S_{s}^{(2) 2}$; a single elastic constant $K$ is assumed, in which $\eta$ is the corresponding viscosity coefficient, $S_{s}^{(2)}$ is the order parameter for the relatively slow motions, and other symbols have their usual meanings. With regard to splay deformations, the so-called bending rigidity is $\kappa \approx K t$, where $t$ is the bilayer thickness, giving a $\kappa^{-3 / 2}$ dependence of $R_{1 Z}$. Moreover, 3D director fluctuations ( $d$ $=3$ ) yield an $\omega^{-1 / 2}$ frequency dispersion as a characteristic signature $[2,15]$. In this case the reduction in the square-law slope, cf. Fig. 3(b), reflects an increase in $\kappa$ and/or $S_{s}^{(2)}$ due to short-range cholesterol-phospholipid interactions. At the molecular level, a dynamical protrusion of cholesterol between the apposed monolayers may occur, as suggested by recent quasielastic neutron scattering studies [18]. 


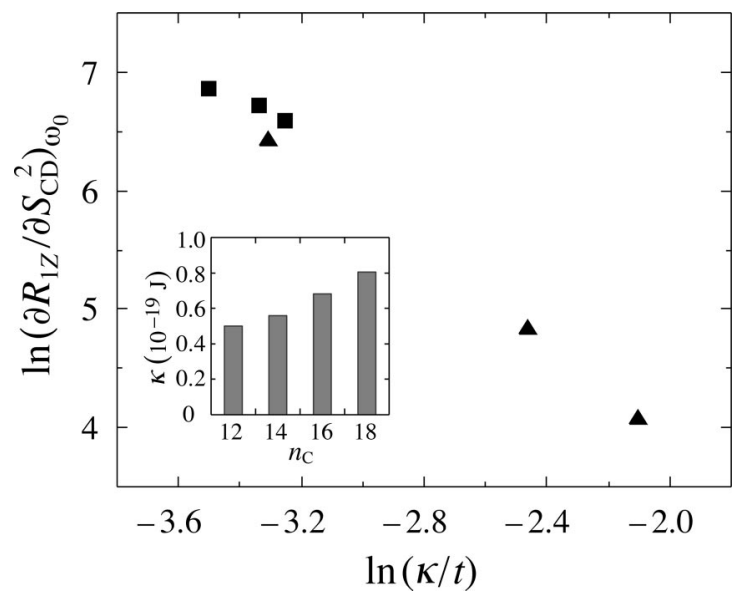

FIG. 4. Comparison of ${ }^{2} \mathrm{H}$ NMR square-law slopes to bending rigidity $\kappa$ obtained from thermal shape fluctuation data for lipid vesicles [5,7]. (覀 Results for homologous series of PC's with acyl carbon lengths of $n_{\mathrm{C}}=12,14$, and 16 in the $L_{\alpha}$ phase at $55.4 \mathrm{MHz}$; (வ) data at $76.8 \mathrm{MHz}$ for DMPC/cholesterol mixtures with $X_{C}$ $=0,0.33$, and 0.50 in the liquid-ordered phase. The inset shows the values of $\kappa$ estimated from the ${ }^{2} \mathrm{H}$ NMR model for the homologous series of PC's.

The correspondence of the NMR relaxation rates to bulk elastic fluctuations of membrane lipids is further born out by comparing the ${ }^{2} \mathrm{H}$ NMR results to available data for the macroscopic bending rigidities of lipid bilayers [4,5,7,19]. Figure 4 shows double-logarithmic plots of the square-law slopes $\left(\partial R_{1 Z} / \partial S_{\mathrm{CD}}^{2}\right)_{\omega_{0}}$ for DMPC- $d_{54} /$ cholesterol mixtures having different cholesterol molar ratios $X_{C}$ versus $\kappa$ determined from video microscopy of large unilamellar vesicles [7]. The values of $\kappa$ are divided by $t$, the bilayer thickness [20]. We also show a comparison for a series of phosphatidylcholines (PC's) in the $L_{\alpha}$ phase with acyl chains ranging in length from $\mathrm{C}_{12}$ to $\mathrm{C}_{16}$ [5]. The observed correlation points to an interpretation in terms of quasi-elastic bilayer deformations. Using data for the DMPC- $d_{54}$ bilayer as a reference [5], one can calculate the bending elasticity for the homologous series of PC's [21] using Eqs. (2) and (3), i.e. assuming a $\kappa^{-3 / 2}$ dependence, as shown in the inset to Fig. 4. The estimated bending rigidity increases by $\approx 60 \%$ going from $C_{12}$ to $C_{18}$ acyl chains based on the ${ }^{2} \mathrm{H}$ NMR model, in agreement with thermal shape fluctuations [5] and micropipette deformation of giant PC vesicles [4].

In summary, nuclear spin-lattice relaxation studies of fluid lipid bilayers manifest their quasi-elastic deformation on short length and time scales, e.g., on the order of the membrane thickness and less [2]. Our interpretation of the atomspecific NMR relaxation data is in broad agreement with MD simulations of flexible surfactant films [8] and lipid membranes [9]. As discussed by Lindahl and Edholm [9], local trans-gauche isomerizations are accompanied by concerted isomerizations about multiple bonds in the assembly of lipid acyl chains. The present continuum model approximates these collective excitations in mathematical closed form, where the influences of cholesterol and the membrane thickness are described in terms of the bilayer bending energy. Finally, the bilayer softness as studied with NMR relaxation may be significant to lipid-protein interactions in fluid membranes [22], where elastic curvature deformation may be an important variable.

This research was supported by the U.S. National Institutes of Health.
[1] S. L. Keller et al., Phys. Rev. Lett. 81, 5019 (1998).

[2] M. F. Brown, J. Chem. Phys. 77, 1576 (1982).

[3] G. Fragneto et al., Europhys. Lett. 53, 100 (2001).

[4] W. Rawicz et al., Biophys. J. 79, 328 (2000).

[5] L. Fernandez-Puente et al., Europhys. Lett. 28, 181 (1994).

[6] E. Sackmann, in Handbook of Biological Physics, edited by R. Lipowsky and E. Sackmann (Elsevier, Amsterdam, 1995), Vol. 1, pp. 213-304.

[7] P. Méléard et al., Biophys. J. 72, 2616 (1997).

[8] R. Goetz, G. Gompper, and R. Lipowsky, Phys. Rev. Lett. 82, 221 (1999).

[9] E. Lindahl and O. Edholm, J. Chem. Phys. 115, 4938 (2001).

[10] W. Helfrich and R.-M. Servuss, Nuovo Cimento D 3, 137 (1984).

[11] D. Anderson, H. Wennerström, and U. Olsson, J. Phys. Chem. 93, 4243 (1989).

[12] M. Bloom, E. Evans, and O. G. Mouritsen, Q. Rev. Biophys. 24, 293 (1991).
[13] M. F. Brown and S. I. Chan, in Encyclopedia of Nuclear Magnetic Resonance, edited by D. M. Grant and R. K. Harris (Wiley, New York, 1996), Vol. 2, pp. 871-885, and references therein.

[14] T. P. Trouard et al., J. Chem. Phys. 110, 8802 (1999).

[15] A. A. Nevzorov, T. P. Trouard, and M. F. Brown, Phys. Rev. E 58, 2259 (1998).

[16] M. Bloom and E. Evans, in Biologically Inspired Physics, edited by L. Peliti (Plenum, New York, 1991), pp. 137-147.

[17] J. Stohrer et al., J. Chem. Phys. 95, 672 (1991).

[18] E. Endress et al., Biochemistry 41, 13078 (2002).

[19] E. Brückner, S. Sonntag, and H. Rehage, J. Phys. Chem. B 104, 2311 (2000).

[20] H. I. Petrache, S. W. Dodd, and M. F. Brown, Biophys. J. 79, 3172 (2000).

[21] M. F. Brown et al., Phys. Rev. E 64, 010901 (2001).

[22] A. V. Botelho et al., Biochemistry 41, 6354 (2002). 